\title{
Analyse des herbicides phénoxyacides par chromatographie en phase liquide : $1^{\text {er }}$ essai inter-laboratoires
}

\section{HPLC analysis of phenoxyacid herbicides : $I^{\text {st }}$ interlaboratory comparison study}

Alain TURCANT ${ }^{(1) * * *}$, Betty DEHON ${ }^{(2) *}$, Catherine GANIÈRE-MONTEIL ${ }^{(3) *}$,
Sylvain DULAURENT ${ }^{(4) *}$, Moustapha MOULSMA ${ }^{(5)}$, Corinne CHARLIER ${ }^{(6) *}$

(1) Pharmacologie-Toxicologie, CHU Angers (2) Biochimie UF Toxicologie, CHU Lille

(3) Pharmacologie, CHU Nantes

(4) Pharmacologie-Toxicologie, CHU Limoges

(5) UF Pharmacotoxicologie, CHU E. Herriot Lyon

(6) Toxicologie Clinique et Médicolégale, CHU Liège

\# Groupe de travail "Pesticides" de la SFTA

*Auteur à qui adresser la correspondance : Alain TURCANT, Service de Pharmacologie et Toxicologie,

CHU, 4, rue Larrey - 49933 ANGERS Cedex 9 - France

Tél : 0241354552 - Fax : 0241354877 -E-mail : alturcant@chu-angers.fr

(Reçu le 16 février 2006 ; accepté le 19 juin 2006)

\section{$R \hat{E} S U M{ }^{\prime}$}

Afin de documenter au mieux, par des dosages plasmatiques, les cas d'intoxication par les herbicides phénoxyacides, six laboratoires ont évalué diverses approches analytiques de type CLHP après purification par simple précipitation des protéines plasmatiques par l'acétonitrile ou après extraction liquide-liquide en milieu acide ou encore après extraction liquide-solide. Un essai comparatif concernant 4 plasmas surchargés et 4 cas réels d'intoxication a montré une variabilité correcte avec un CV la plupart du temps inférieur à 20\%. L'extraction liquide-solide, non totalement satisfaisante dans cet essai, nécessite une investigation plus poussée tandis que la simple précipitation protéique apporte une

\section{SUMMARY}

The evaluation of acute phenoxyacid poisonings in humans requires the identification of the herbicide and, if possible, its quantification in plasma. Six laboratories tested different analytical approaches, using HPLC, after sample treatment either by simple protein precipitation or by liquid-liquid extraction under acidic pH conditions or by solid phase extraction. A comparative study on 4 spiked plasmas and 4 real acute cases showed good results with $C V<20 \%$. Solid phase extraction needs further investigation while simple precipitation by acetonitrile provides a rapid and sufficient cleaning procedure to evaluate the majority of acute poisonings. Liquid-liquid extraction, providing lower limits of 
bonne rapidité de préparation des échantillons et une sensibilité suffisante pour la majorité des cas d'intoxication aiguë. L'extraction liquide-liquide permet une meilleure sensibilité, notamment avec une détection par spectrométrie de. masse en tandem et rend cette approche applicable aux cas d'intoxication faible voire aux expositions professionnelles.

\section{MOTS-CLÉS}

Phérnoxyacides, phytohomones, herbicides, intoxication, plasma, CLHP.

\section{Introduction}

Les herbicides phénoxyacides (fig.1a) constituent une famille de produits phytosanitaires agissant de façon systémique par pénétration foliaire en stimulant de façon anarchique la croissance de la plante. Sept substances sont commercialisées en France : cinq sont de type acide phénoxy-acétique, avec différentes substitutions par un ou plusieurs atomes de chlore $(\mathrm{Cl})$ ou un groupement méthyle (CH3), deux d'entre elles présentant un groupement méthyle sur la chaîne alcanoïque, d'où leur autre appellation d'acides phénoxy-propioniques, et 2 sont des acides phénoxy-butyriques (Tableau I). Le fénoprop ou 2,4,5-TP, également appelé silvex, non commercialisé en France mais référencé en Belgique comme devant être recherché dans les eaux de rivières, complète cette liste. Le triclopyr, où le cycle phényle est remplacé par un noyau pyridine, est apparenté à cette famille (fig.1b). Les pKa diffèrent légèrement d'une substance à l'autre en fonction de la longueur de la chaîne acide ou de la substitution par un ou plusieurs $\mathrm{Cl}$ ou un groupement méthyle. Ces produits sont souvent commercialisés en association par deux ou encore avec d'autres molécules comme le dicamba (herbicide de la famille des dérivés de l'acide benzoïque). Ces herbicides agissent comme l'Acide Indole Acétique (IAA) qui est une auxine naturelle et sont donc également appelés auxines de synthèse ou encore phytohormones (1). L'analogie structurale avec 1'IAA (fig.2) est basée sur 3 similitudes:

- une partie plane (cycles aromatiques),

- la présence d'une fonction acide carboxylique

- l'existence d'une structure dipolaire entre le groupement acide et une zone électrophile, azote du noyau indole ou carbone en position 2 du cycle. En raison de l'effet électro-attracteur des halogènes, la distance entre les charges est voisine de 5,5 angströms (1).
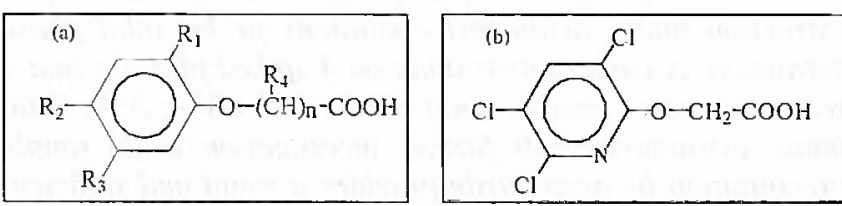

Figure 1 : Formules chimiques des phénoxyacides $(a)$ et triclopyr (b). detection especially when tandem mass spectronetry is used, will be more interesting in minor or professional exposition to these herbicides.

\section{KEY-WORDS}

Phenoxyacids, herbicides, acute poisoning, plasma, HPLC.

Tableau I : Dénomination, substituants, pKa et masses molaires des différents phénoxjacides et apparentés (cfformules figure 1).

\begin{tabular}{|l|c|c|c|c|c|c|c|}
\hline Ac Phérioxy- & $\mathrm{R} 1$ & $\mathrm{R} 2$ & $\mathrm{R} 3$ & $\mathrm{R} 4$ & $\mathrm{n}$ & $\mathrm{pK}$ & $\mathrm{MM}$ \\
\hline acétiques & & & & & & & \\
2,4-D (2,4-dichloro-) & $\mathrm{Cl}$ & $\mathrm{Cl}$ & $\mathrm{H}$ & $\mathrm{H}$ & 1 & 2,64 & 221,0 \\
2,4,5-T (2,4,5-trichloro-) & $\mathrm{Cl}$ & $\mathrm{Cl}$ & $\mathrm{Cl}$ & $\mathrm{H}$ & 1 & 2,80 & 255,5 \\
MCPA (2-méthyl-4-chloro-) & $\mathrm{CH} 3$ & $\mathrm{Cl}$ & $\mathrm{H}$ & $\mathrm{H}$ & $\mathrm{I}$ & 3,07 & 200,6 \\
\hline propioniques & & & & & & & \\
2,4-DCP (Dichlorprop) & $\mathrm{Cl}$ & $\mathrm{Cl}$ & $\mathrm{H}$ & $\mathrm{CH} 3$ & 1 & 3,00 & 235,1 \\
2,4,5-TP (Fénoprop) & $\mathrm{Cl}$ & $\mathrm{Cl}$ & $\mathrm{Cl}$ & $\mathrm{CH} 3$ & 1 & 2,80 & 269,5 \\
MCPP (Mécoprop) & $\mathrm{CH} 3$ & $\mathrm{Cl}$ & $\mathrm{H}$ & $\mathrm{CH} 3$ & $\mathrm{I}$ & 3,78 & 214,6 \\
\hline butyriques & & & & & & & \\
2,4-DB (2,4-dichloro-) & $\mathrm{Cl}$ & $\mathrm{Cl}$ & $\mathrm{H}$ & $\mathrm{H}$ & 3 & 4,80 & 249,1 \\
MCPB (2-méthyl-4-chloro-) & $\mathrm{CH} 3$ & $\mathrm{Cl}$ & $\mathrm{H}$ & $\mathrm{H}$ & 3 & 4,84 & 228,7 \\
\hline Triclopyr (cycle pyridine) & $\mathrm{Cl}$ & $\mathrm{Cl}$ & $\mathrm{Cl}$ & $\mathrm{H}$ & 1 & 2,68 & 256,5 \\
\hline
\end{tabular}
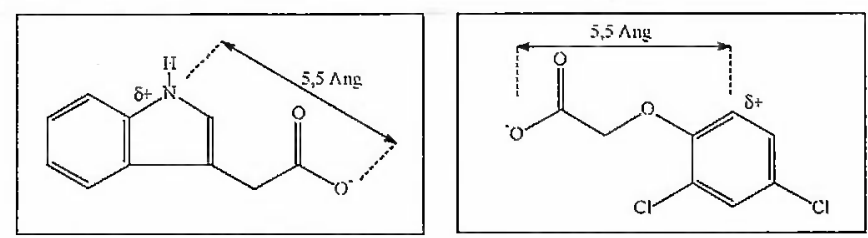

Figure 2 : Analogie géométrique entre l'acide indole acétique et l'acide 2,4-D.

Si la toxicité par absorption cutanée est faible, il n'en est pas de même lors d'ingestion orale puisque les doses potentiellement mortelles sont évaluées entre 80 et $800 \mathrm{mg} / \mathrm{kg}$ selon les substances, soit quelques grammes (2). Les principaux signes cliniques en cas d'intoxication aiguë sont les vomissements précoces, les troubles de la conscience, l'hypotension artérielle et l'acidose métabolique.

Les méthodes de dosage plasmatique et/ou urinaire de ces herbicides phénoxyacides, décrites dans la littérature en cas d'intoxication, sont essentiellement basées sur la chromatographie en phase liquide avec détection UV $(3,4)$ plus facile à mettre en oeuvre que la chromatographie en phase gazeuse qui nécessite une étape de méthylation (5).

Le groupe de travail «Pesticides 》 de la Société Française de Toxicologie Analytique s'est intéressé au suivi des intoxications par herbicides phénoxyacides en 
évaluant les capacités analytiques de six laboratoires, tant d'un point de vue qualitatif que quantitatif, soit par la mise en place d'une méthode analytique nouvelle soit par une réévaluation de sa propre méthode de dosage vis-à-vis de 9 substances. La démarche initiale a été, pour chaque laboratoire, de préciser les limites de détection de ces produits avec sa propre méthode de screening toxicologique par chromatographie en phase liquide avec détection UV à barrette de diodes (CLHP/BD) après extraction alcaline, méthode mise en œuvre dans la plupart des laboratoires pour la recherche des substances en cause au cours d'intoxications aiguës médicamenteuses. L'extraction en milieu alcalin est a priori défavorable pour la mise en évidence des herbicides phénoxyacides. Ensuite, chaque laboratoire participant devait établir des conditions optimisées de recherche et dosage de ces produits en s'aidant éventuellement d'une démarche analytique de type CLHP/BD, proposée par le laboratoire d'Angers, mais chacun disposait du libre choix tant pour l'aspect chromatographique que pour la détection ou la préparation de l'échantillon par extraction liquide/liquide ou liquide/solide ou encore par simple précipitation des protéines. Enfin un essai inter-laboratoires a été réalisé sur 8 échantillons plasmatiques correspondant à 4 plasmas surchargés et à 4 cas réels d'intoxication.

\section{Matériel et Méthodes}

Les poudres, obtenues chez Aldrich, Fluka et Riedel de Haën (Sigma-Aldrich, Saint Quentin Fallavier, France), chez Cluzeau (Ste Foy la Grande, France) ou LGC Promochem (Molsheim, France), sont de pureté comprise entre 95 et $99,9 \%$ selon les produits. Les solutions mères sont préparées, selon les substances, soit à 1 , à 5 ou à $10 \mathrm{~g} / \mathrm{L}$ dans l'acétonitrile (pour analyses) et non le méthanol pour minimiser le risque d'estérification possible in vitro à long terme.

La limite de détection pour chacun de ces produits a été estimée, pour chaque laboratoire, par l'obtention d'un spectre UV interprétable, en surchargeant des plasmas témoins à des concentrations allant de 10 à $100 \mathrm{mg} / \mathrm{L}$ et en testant ses propres conditions d'extraction liquideliquide en milieu alcalin.

La méthode proposée pour l'optimisation du dosage des herbicides fait appel à une prise d'essai de $100 \mu \mathrm{l}$ de plasma auquel est ajouté un volume équivalent d'acétonitrile contenant l'acide 2-méthyl-4-chlorophénoxybutyrique (MCPB) utilisé comme étalon interne à 300 $\mathrm{mg} / \mathrm{L}$, cette substance n'ayant jamais été rencontrée dans les cas d'intoxication dans l'expérience angevine. Le surnageant de centrifugation est ensuite dilué au $1 / 2$ dans l'eau puis analysé par chromatographie de parta- ge en phases inversées soit dans les conditions du screening classique de ce laboratoire (6), c'est-à-dire un gradient tampon phosphate $\mathrm{pH} 6$ et acétonitrile avec une détection à 3 longueurs d'onde $(230,285$ et $210 \mathrm{~nm}$ ), soit par une élution isocratique en tampon formiate $2 \mathrm{mM}$ à $\mathrm{pH} 3$ et acétonitrile $67 / 33 \mathrm{v} / \mathrm{v}$ pour pallier une insuffisance de séparation de certains produits dans les premières conditions. Un exemple de gamme d'étalonnage entre 50 et $500 \mathrm{mg} / \mathrm{L}$ pour les 6 produits habituellement testés est présenté dans la figure 3 . Les limites de quantification sont de $10 \mathrm{mg} / \mathrm{L}$.

L'essai inter-laboratoires proposé comportait 4 plasmas témoins surchargés ( $800 \mu \mathrm{l}$ pour chaque laboratoire) et 4 plasmas (300 à $1000 \mu \mathrm{l}$ ) correspondaient à des cas réels stockés en sérothèque à $-20^{\circ} \mathrm{C}$. Certains de ces échantillons dataient de plus de 10 ans. Les surcharges ont été effectuées par ajout sur $5 \mathrm{ml}$ de plasma témoin d'un volume maximum de $200 \mu \mathrm{l}$ d'acétonitrile contenant une ou deux molécules à différentes concentrations. Les plasmas ont été envoyés congelés et chaque laboratoire disposait de 2 mois pour répondre à cet essai.

Les conditions analytiques utilisées par chaque laboratoire sont rapportées dans le tableau II : 3 ont choisi la précipitation par acétonitrile, 2 l'extraction liquide/liquide en milieu très acide $(\mathrm{pH}<1)$ et un l'extraction liquide/solide sur cartouche échangeuse d'anions (OASIS® MAX) à partir de $100 \mu \mathrm{l}$ de prise d'essai. La séparation chromatographique est réalisée sur colonne $\mathrm{C} 8$ ou $\mathrm{C} 18$ éventuellement avec faible diamètre interne (2 voire $1 \mathrm{~mm}$ ) avec des tampons phosphate ou formiate de concentration et de $\mathrm{pH}$ variés, 4 laboratoires ayant des conditions de gradient et 2 des conditions isocratiques avec des temps de séparation compris entre 15 et $30 \mathrm{~min}$. Un laboratoire a identifié et dosé les phénoxyacides par spectrométrie de masse tandem, les autres utilisant l'UV barrette de diodes avec une ou plusieurs longueurs d'onde. Cinq laboratoires ont quantifié avec l'aide d'un étalon interne, 1 par étalonnage externe, ce laboratoire ayant procédé à l'identification initiale par CPG/ SM après méthylation.

\section{Résultats}

Les résultats des analyses dans les conditions de screening montrent évidemment la faible détectabilité de ces molécules acides dans des conditions d'extraction alcaline même si, compte tenu des concentrations importantes rencontrées en intoxication, il n'est pas impossible de les mettre en évidence. Selon le pH et le solvant d'extraction on observe une grande disparité de détection. Une extraction de plasma à $\mathrm{pH} 11$ par le dichlorométhane ne permet pas de détecter ces produits à la concentration de $100 \mathrm{mg} / \mathrm{l}$. Une extraction en présence 

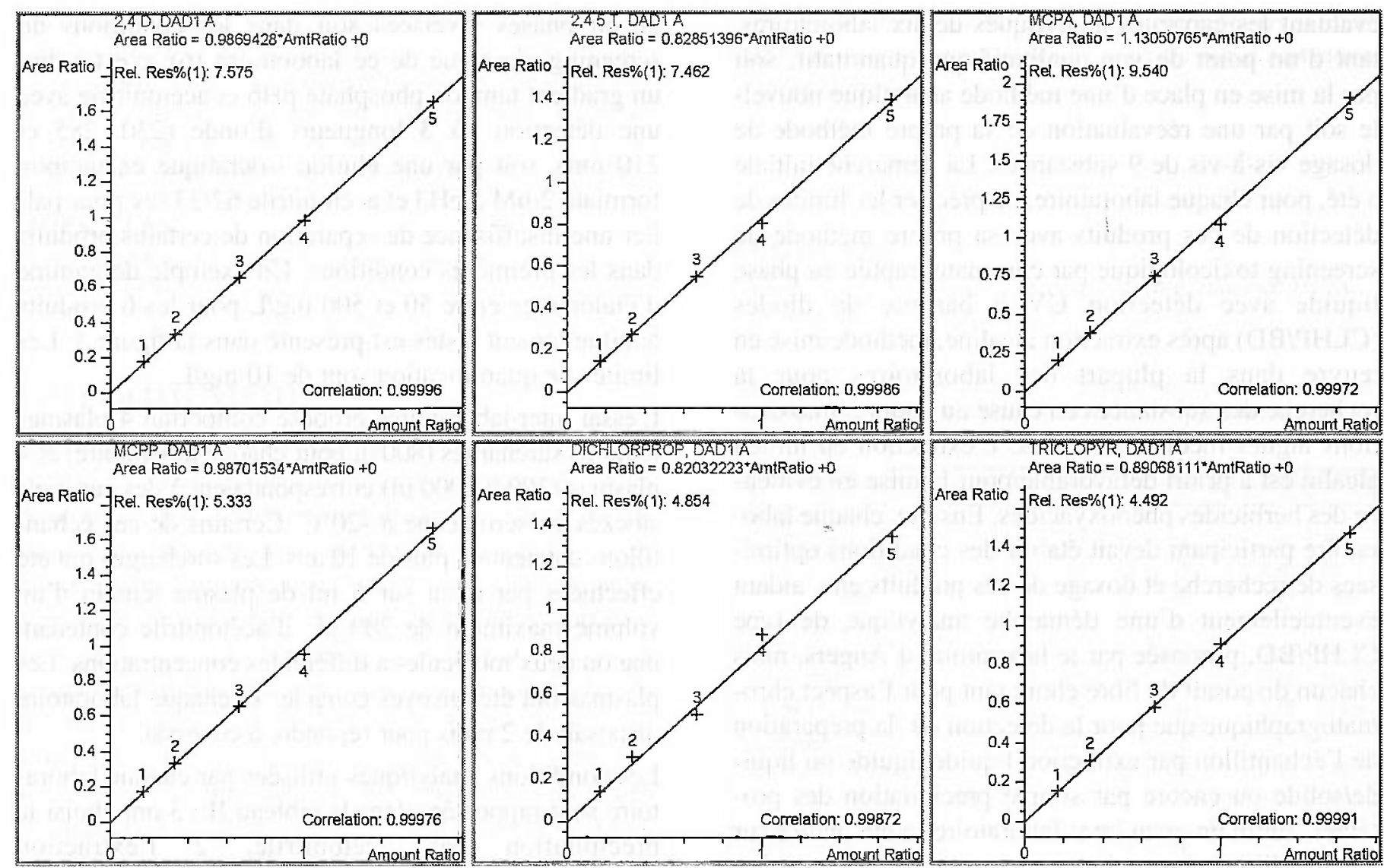

Figure 3: Courbes d'étalonnage de 6 phénoxyacides entre 50 et $500 \mathrm{mg} / \mathrm{L}$ après simple précipitation des protéines plasmatiques $(E I=M C P B 300 \mathrm{mg} / L)$.

Tableau II : Conditions analytiques utilisées par les différents laboratoires.

\begin{tabular}{|c|c|c|c|c|c|c|}
\hline & $\mathbf{L 1}$ & $\mathrm{L} 2$ & $\mathrm{~L} 3$ & $\mathrm{~L} 4$ & 1.5 & L6 \\
\hline P. & $\begin{array}{l}\text { CH3CN } \\
1 / 1 \mathrm{v} / \mathrm{ve}\end{array}$ & $\begin{array}{l}\mathrm{HCl} \text { 1N } \\
1 / 2 \mathrm{v} / \mathrm{ve} \\
\text { Ether } \\
8 / 1 \mathrm{v} / \mathrm{ve}\end{array}$ & $\begin{array}{c}\text { Oasis }^{*} \mathrm{MAX} \\
\mathrm{L}: \mathrm{MeOH} \\
\mathrm{E}: \mathrm{MeOH} / \mathrm{ac} \\
\text { formique } 98 / 2\end{array}$ & $\begin{array}{l}\mathrm{HCl} 6 \mathrm{~N} \\
1 / 4 \mathrm{v} / \mathrm{ve} \\
\mathrm{AcEt} \\
4 / 1 \mathrm{v} / \mathrm{ve}\end{array}$ & $\begin{array}{l}\text { CH3CN } \\
1 / 1 \mathrm{v} / \mathrm{ve}\end{array}$ & $\begin{array}{l}\text { CH3CN } \\
1 / 1 \mathrm{v} / \mathrm{ve}\end{array}$ \\
\hline C. & $\begin{array}{c}\text { C18 } \\
\text { Hypersil } \\
\text { di } 2,1 \mathrm{~mm}\end{array}$ & $\begin{array}{c}\text { C8 } \\
\text { Symmetry }\end{array}$ & $\begin{array}{c}\text { C18 } \\
\text { Eclipse }\end{array}$ & $\begin{array}{c}\text { C18 } \\
\text { Nucleosil } \\
\text { di } 1 \mathrm{~mm}\end{array}$ & $\begin{array}{c}\text { C18 } \\
\text { Atlantis } \\
\text { di } 2,1 \mathrm{~mm}\end{array}$ & $\begin{array}{c}\text { C8 } \\
\text { Symmetry }\end{array}$ \\
\hline S. & $\begin{array}{c}\text { P } 20 \mathrm{mM} \\
\text { pH } 6 \\
\text { CH3CN } \\
\text { G } \\
18 \mathrm{~min}\end{array}$ & $\begin{array}{c}\text { P } 44 \mathrm{mM} \\
\text { pH } 3,8 \\
\text { CH3CN } \\
\text { G } \\
30 \mathrm{~min}\end{array}$ & $\begin{array}{l}\mathrm{F} 5 \mathrm{mM} \\
\mathrm{pH} 3 \\
\mathrm{CH} 3 \mathrm{CN} \\
\mathrm{I} 70 / 30 \\
15 \mathrm{~min}\end{array}$ & $\begin{array}{c}\mathrm{F} 2 \mathrm{mM} \\
\mathrm{pH} 3 \\
\mathrm{CH} 3 \mathrm{CN} \\
\mathrm{G} \\
18 \mathrm{~min}\end{array}$ & $\begin{array}{l}\text { F } 5 \mathrm{mM} \\
\mathrm{pH} 6 \\
\mathrm{CH} 3 \mathrm{CN} \\
\text { I 67/33 } \\
30 \mathrm{~min}\end{array}$ & $\begin{array}{c}\mathrm{P} 50 \mathrm{mM} \\
\mathrm{pH} \mathrm{3,6} \\
\mathrm{CH} 3 \mathrm{CN} \\
\mathrm{G} \\
25 \mathrm{~min}\end{array}$ \\
\hline D. & $\begin{array}{c}\text { UV-BD } \\
(230,285,210) \\
\text { EI : MCPB }\end{array}$ & $\begin{array}{c}\text { UV-BD } \\
(230) \\
\text { EI : Prazépam }\end{array}$ & $\begin{array}{c}\text { UV-BD } \\
(230) \\
\text { EI : MCPB }\end{array}$ & $\begin{array}{c}\text { SM/SM } \\
\text { MRM } \\
\text { (2 ions) } \\
\text { EI : Méphénytoinne }\end{array}$ & $\begin{array}{c}\mathbf{U V}-\overline{B D} \\
(230,200) \\
\text { EE }\end{array}$ & $\begin{array}{c}\text { UV-BD } \\
(220) \\
\text { EI : MCPB }\end{array}$ \\
\hline$\overline{L Q}$ & $10 \mathrm{mg} / \mathrm{L}$ & $0,2 \mathrm{mg} / \mathrm{L}$ & 1 à $10 \mathrm{mg} / \mathrm{L}$ & 20 à $50 \mu \mathrm{g} / \mathrm{L}$ & & \\
\hline
\end{tabular}

(P. : préparation échantillon; v/ve volume réactif/volume échantillon ; L. lavage ; E. élution ; C. colonne ; di diamètre interne ; S. solvant ; P. phosphate ; F. formiate ; G gradient ; I isocratique ; D. détection ; BD barrette de diodes, longueurs d'onde de quantification entre parenthèses ; SM/SM spectrométrie de masse en tandem; MRM multiple reaction monitoring ; étalonnage interne EI ou externe EE; LQ limite de quantification).

de carbonate $1 \mathrm{M}(2 / 1 \mathrm{v} / \mathrm{v})$ par un solvant quaternaire éther/dichlorométhane/hexane/alcool isoamylique $50 / 30 / 20 / 0,5 \mathrm{v} / \mathrm{v} / \mathrm{v} / \mathrm{v}$ permet de détecter les herbicides entre 10 et $100 \mathrm{mg} / \mathrm{L}$ selon le pKa du produit. Une extraction à $\mathrm{pH} 9,5$ en milieu $\mathrm{NH} 4 \mathrm{Cl}$ saturé par un mélange ternaire chloroforme/isopropanol/heptane $60 / 14 / 26 \mathrm{v} / \mathrm{v} / \mathrm{v}$ permet à deux laboratoires une détection proche de $10 \mathrm{mg} / \mathrm{L}$, quelles que soient les molécules. 
Les plasmas surchargés (P1 à P4) contenaient soit une seule molécule, le triclopyr (P1) ou un mélange de 2,4$\mathrm{D}$ et DCP (P2), un mélange 2,4,5-T et fénoprop (P3) ou encore un mélange MCPA et MCPP (P4). Le chromatogramme obtenu par l'un des laboratoires, après extraction par l'éther, est présenté dans la figure 4. Les spectres UV de ces différentes substances (fig.5) présentent des analogies spectrales avec, pour tous, une

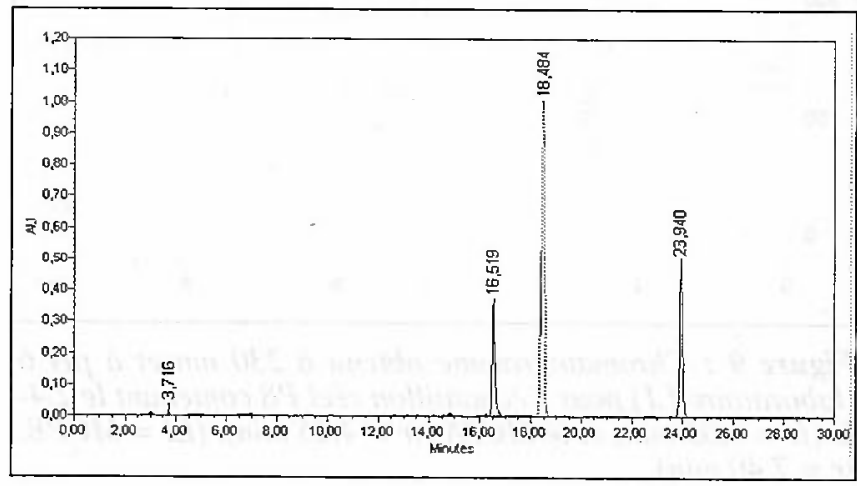

Figure 4: Chromatogramme obtenu à $230 \mathrm{~nm}$ (laboratoire L2) après extraction acide par l'éther de l'échantillon P3 contenant l'acide 2,4,5-T à $75 \mathrm{mg} / \mathrm{L}(\mathrm{tr}=16,5 \mathrm{~min})$ et le fénoprop $(2,4,5-T P)$ à $250 \mathrm{mg} / \mathrm{L}(\mathrm{tr}=18,5 \mathrm{~min})$. $(E I=\mathrm{pra}$ zépam, $t r=23,9 \mathrm{~min})$. longueur d'onde maximale ou un épaulement à 230 nm, ainsi qu'un $2^{\circ}$ maximum entre 280 à $300 \mathrm{~nm}$ selon le nombre de $\mathrm{Cl}$. Ces analogies sont plus importantes lorsqu'il y a un même type de substitution $(2 \mathrm{Cl}, 3 \mathrm{Cl}$ ou $1 \mathrm{Cl}-1 \mathrm{Me})$, le spectre du triclopyr montrant une intensité des maxima très différente en raison du cycle pyridine. L'identification ne pourra donc complètement se faire sur le simple spectre mais nécessite une séparation chromatographique satisfaisante pour un maximum de spécificité.

Les plasmas issus des cas réels P5, P6 et P7 (fig. 6-8) contenaient respectivement du triclopyr; du 2,4-D et MCPP ou du 2,4-D et 2,4,5-T. Le dernier échantillon P8 (fig.9) contenait un mélange 2,4-D et MCPA, non séparés dans les conditions d'élution à pH 6, comme on peut le voir dans la superposition spectrale et dans la comparaison du spectre hybride et de celui de chacun des produits (fig.10a et b). Mais ceux-ci sont séparés à pH 3 (fig.11).

Les résultats quantitatifs (Tableau III) montrent une assez bonne homogénéité par rapport aux valeurs ajoutées avec, pour 4 produits sur 7 un $\mathrm{CV}<20 \%$. Cependant 9 sur 42 valeurs, soit $20 \%$ des résultats, s'écartent de plus de $20 \%$ de la valeur moyenne et les extrêmes peuvent aller du simple au double. Les résultats sur les cas réels (Tableau IV) sont plutôt meilleurs

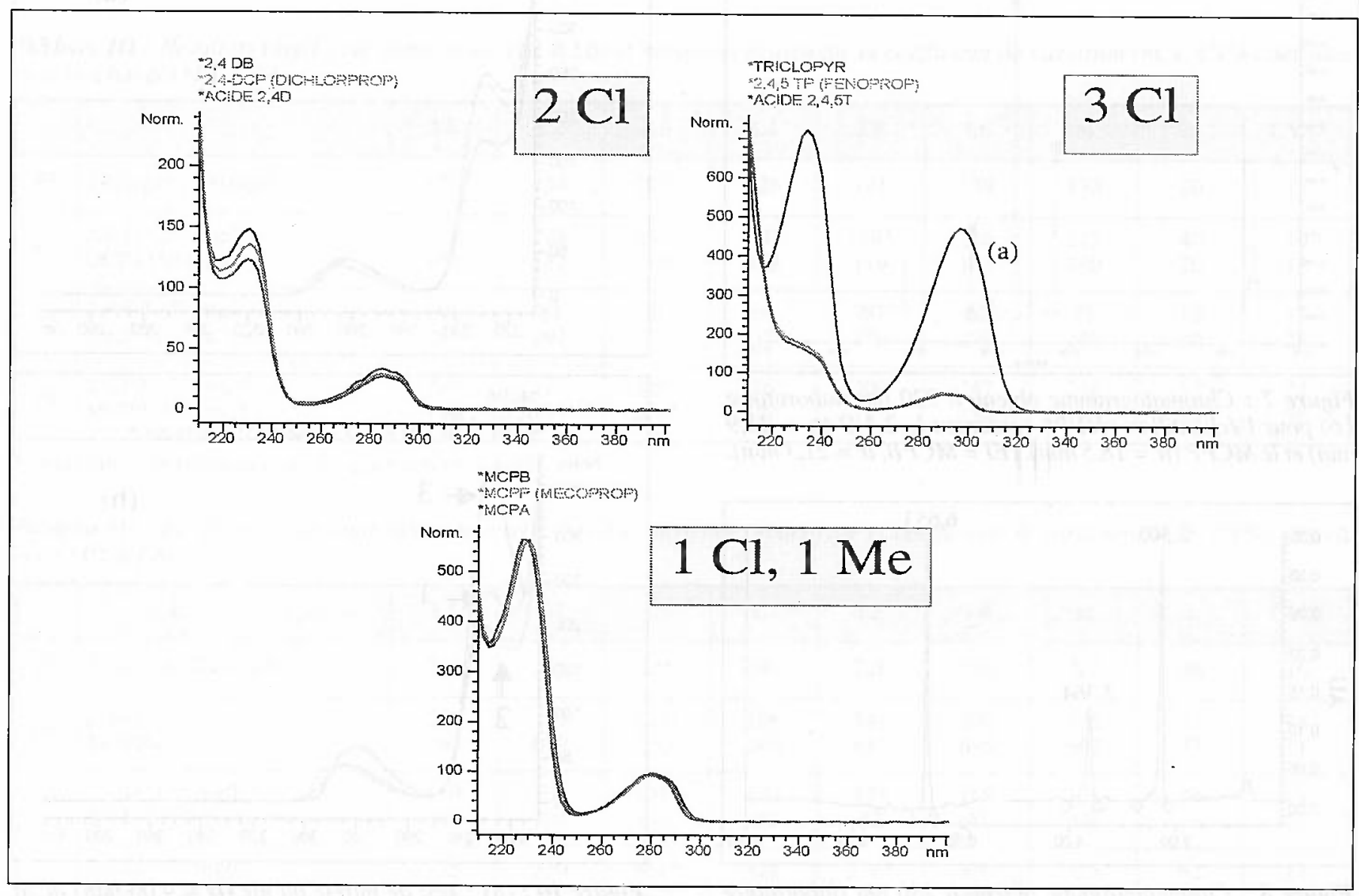

Figure 5 : Spectres UV obtenus à pH 6 entre 210 et 400 nm des phénoxyacides et apparentés : (2C1) dérivés dichlorés; (3C1) dérivés trichlorés dont le triclopyr (a); $(1 \mathrm{CI}, \mathrm{IMe})$ dérivés chloro-et méthyl-. 
avec des CV tous inférieurs à $20 \%$ et seulement 7 résultats en dehors de $\pm 20 \%$. Les valeurs trouvées sont voisines de celles initialement rendues par le laboratoire angevin, montrant une bonne stabilité à $-20^{\circ} \mathrm{C}$. Le plasma 6 était un mélange de 3 prélèvements avec des valeurs voisines de 150 à $200 \mathrm{mg} / \mathrm{L}$ pour le 2,4-D et de 600 à $700 \mathrm{mg} / \mathrm{L}$ pour le MCPP.

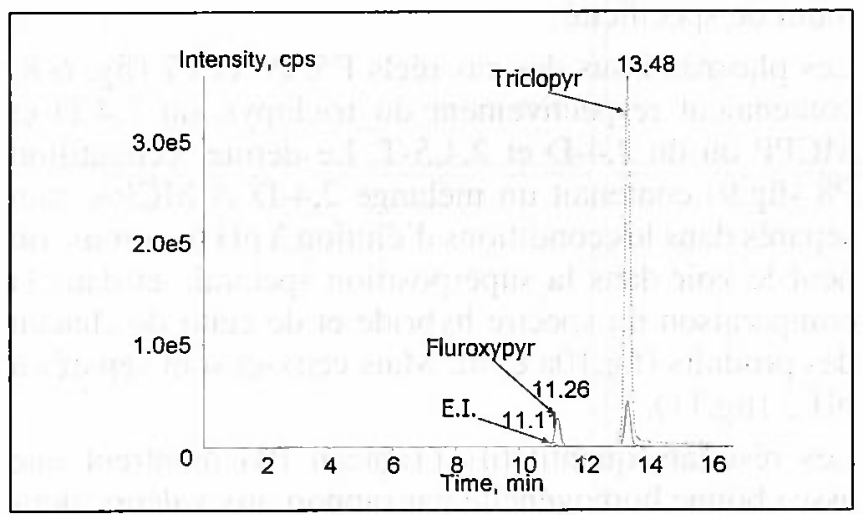

Figure 6: Chromatogramme obtenu en CLHP/SM/SM pour l'échantillon réel $P 5$ contenant le triclopyr $(\mathrm{tr}=13,5 \mathrm{~min}$, $M R M$ 254/196) et le fluroxypyr $(\mathrm{tr}=11,3 \mathrm{~min}, M R M$ 253/195). $(E I=$ méphénytoine, $t r=11,1 \mathrm{~min}, \quad M R M$ $217 / 137,9)$.

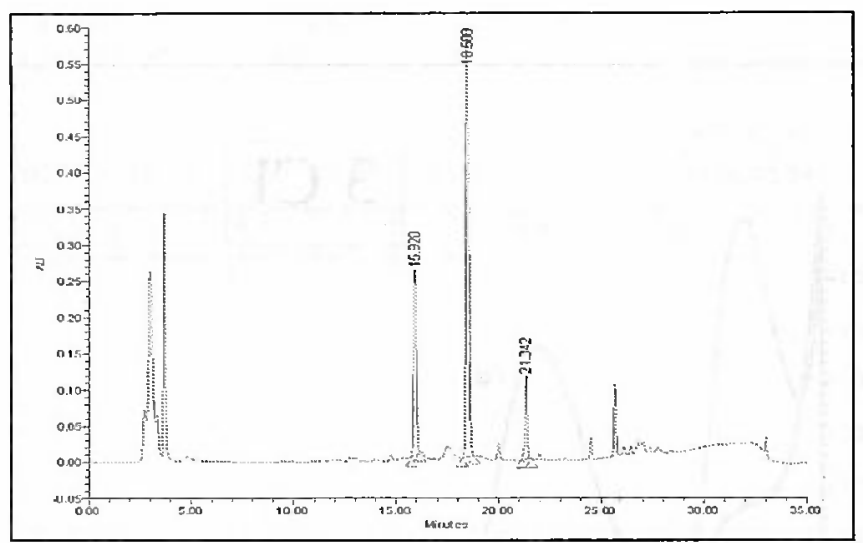

Figure 7 : Chromatogramme obtenu à 220 nm (laboratoire L6) pour l'échantillon réel $P 6$ contenant le 2,4-D $(t r=15,9$ min) et le $M C P P(t r=18,5 \mathrm{~min})$. $(E I=M C P B, t r=21,3 \mathrm{~min})$.

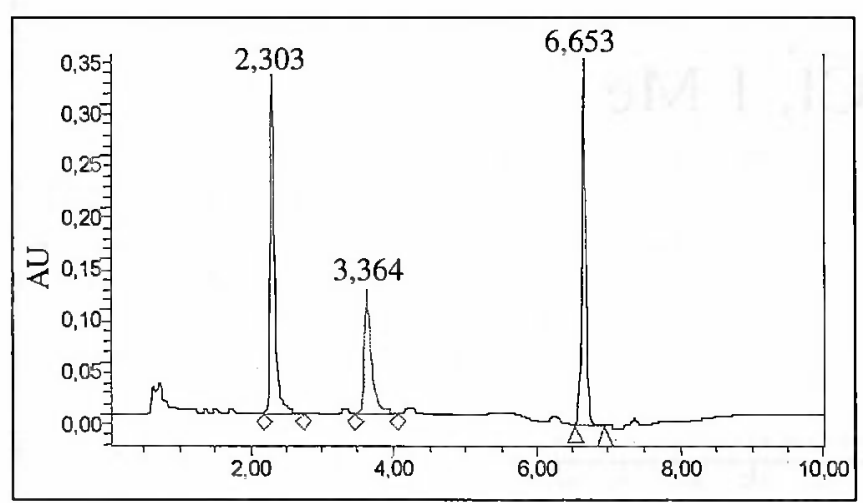

Figure 8: Chromatogramme obtenu à $230 \mathrm{~nm}$ (laboratoire L3) pour l'échantillon réel $P 7$ contenant le $2,4-D(t r=2,30$ min) et le 2,4,5-T $(t r=3,63 \mathrm{~min}) .(E I=M C P B, t r=6,65 \mathrm{~min})$.

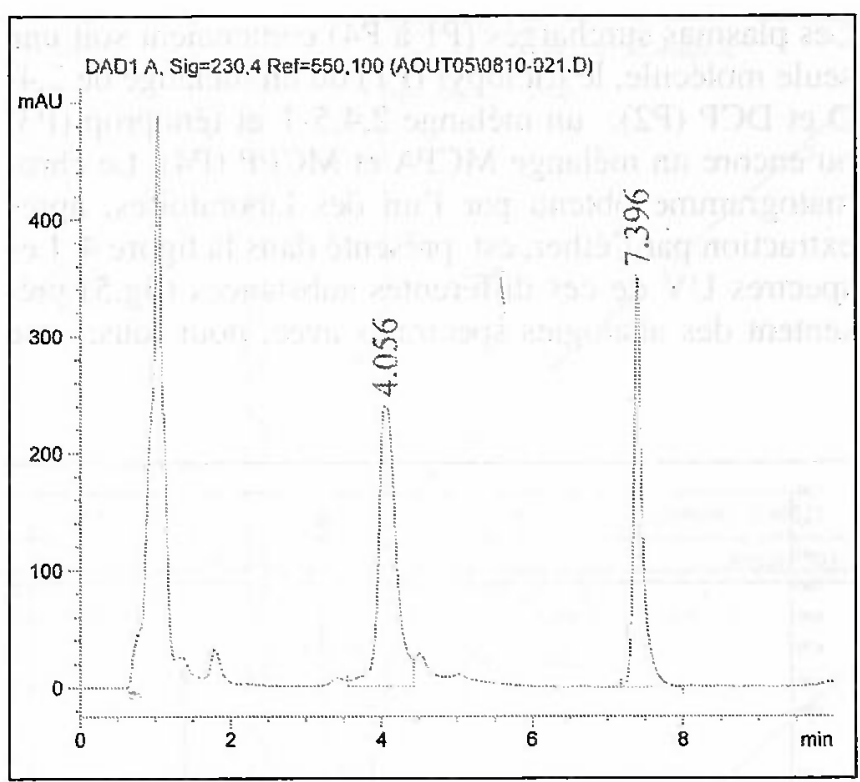

Figure 9 : Chromatogranme obtenu à $230 \mathrm{~nm}$ et à $\mathrm{pH} 6$ (laboratoire L1) pour l'échantillon réel $P 8$ contenant le 2,4$D(t r=4,05 \mathrm{~min})$ et le $M C P A(t r=4,05 \mathrm{~min})$. $(E I=M C P B$, $\mathrm{tr}=7,40 \mathrm{~min})$.

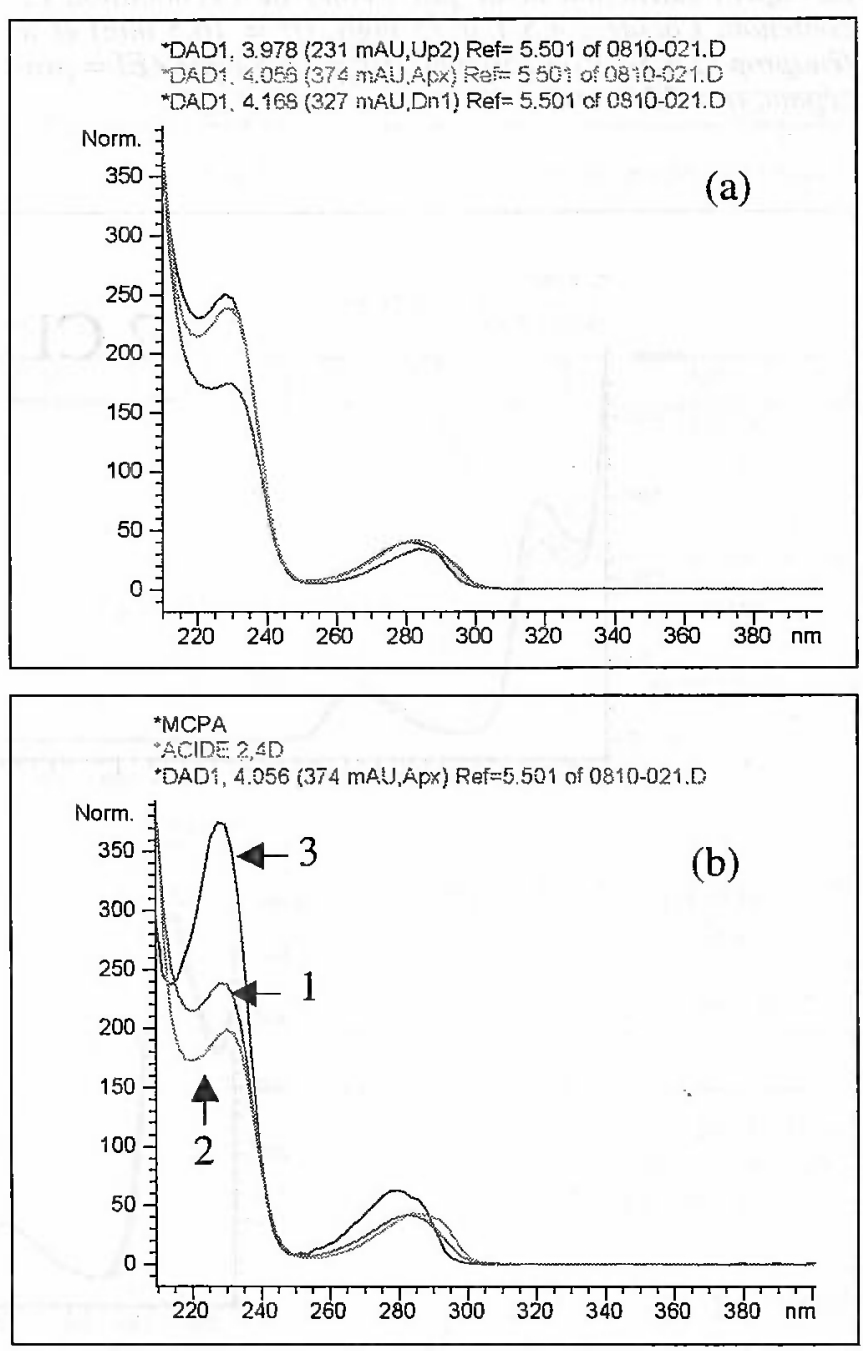

Figure $10:(a):$ Test de pureté du pic $(t r=4,06 \mathrm{~min})$ de la figure $9:(b)$ : comparaison entre spectre hybride au sommet (1) et spectres du 2,4-D (2) et du MCPA (3). 


\section{Discussion}

En raison du caractère acide faible des herbicides phénoxyacides, les procédures classiques d'extraction en milieu alcalin, mises en œuvre dans les méthodes de screening toxicologique, ne sont pas complètement adaptées à la détection de ces substances, surtout en cas

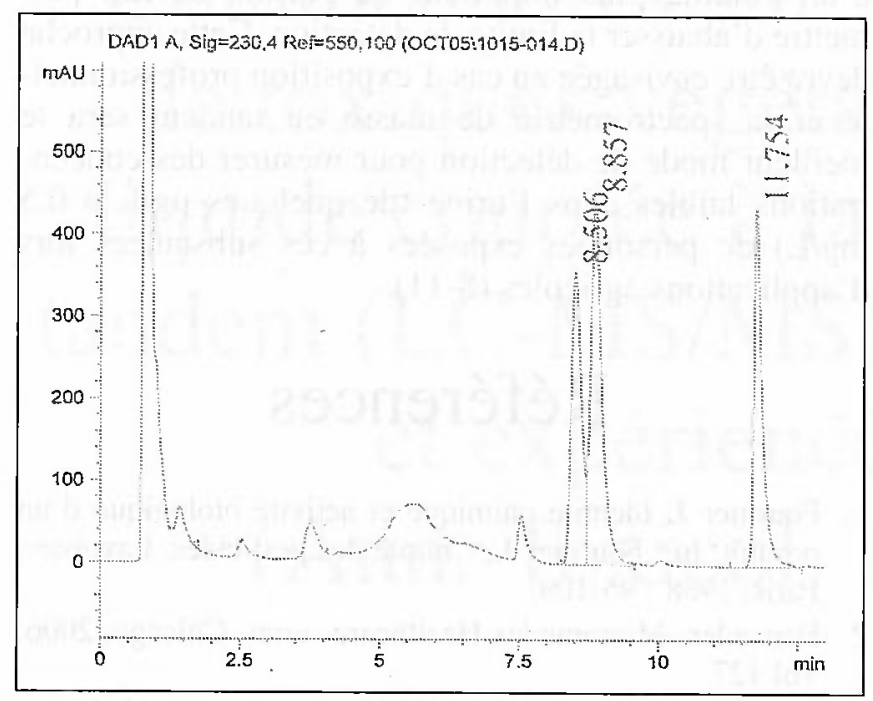

Figure 11 : Séparation chromatographique à pH 3 (laboratoive L1) de l'échantillon réel $P 8: 2,4-D(t r=8,51$ min $)$ et $M C P A(t r=8,56 \mathrm{~min}) .(E I=M C P B, t r=11,75 \mathrm{~min})$. d'intoxication mineure. Il est donc nécessaire de disposer d'une méthode de dosage spécifique pour ces herbicides même si les intoxications sévères, caractérisées par des concentrations plasmatiques de quelques centaines de $\mathrm{mg} / \mathrm{L}$, sont généralement mises en évidence par ces conditions de screening toxicologique.

Parmi les 7 molécules présentes dans l'essai, deux n'ont pas été correctement identifiées dans un premier temps par deux laboratoires car ces substances n'étaient pas référencées dans leur méthode : dans un cas, le triclopyr a été confondu avec le MCPA malgré un spectre UV a priori différent et dans l'autre cas, les transitions SM/SM du fénoprop n'avaient pas été incluses dans la table d'acquisition. Après actualisation des méthodes, chacun a pu procéder au dosage. Pour un laboratoire, l'identification et le dosage du plasma P8 ont nécessité un changement de phase mobile en raison de la coélution du 2,4-D et du MCPA à pH 6.

Les résultats des 8 essais ont montré une bonne homogénéité pour les laboratoires ayant utilisé la simple précipitation des protéines ou l'extraction liquide-liquide. Cependant le laboratoire ayant utilisé l'extraction liquide-solide a présenté plusieurs valeurs en dehors de \pm $20 \%$. Cette approche serait-elle moins performante pour ces produits ou une amélioration de la procédure est-elle envisageable ? Plusieurs questions peuvent être soule-

Tableau III : Résultats (mg/L) par laboratoire (Ll à L6) et moyenne, écart-type et coefficient de variation (m, s, CV\%) des plasmas surchargés (PI à P4).

\begin{tabular}{|l|l|c|c|c|c|c|c|c|c|c|}
\hline & & L1 & L2 & L3 & L4 & L5 & L6 & m & s & CV\% \\
\hline P1 & Triclopyr (150 mg/L) & 155 & 154 & $103^{*}$ & 126 & 121 & 139 & 133 & 20 & 15 \\
\hline P2 & $2,4-\mathrm{D}(250 \mathrm{mg} / \mathrm{L})$ & 255 & 228 & $154^{*}$ & 207 & $188^{*}$ & 256 & 215 & 40 & 19 \\
& DCP (150 mg/L) & 156 & 152 & $110^{*}$ & 158 & 119 & 143 & 140 & 20 & 15 \\
\hline \multirow{2}{*}{ P3 } & $2,4,5-\mathrm{T}(75 \mathrm{mg} / \mathrm{L})$ & 75 & 82 & 89 & 60 & 60 & 67 & 72 & 12 & 17 \\
& $2,4,5-\mathrm{TP}(250 \mathrm{mg} / \mathrm{L})$ & 265 & 292 & $173^{*}$ & $155^{*}$ & 276 & 279 & 240 & 60 & 25 \\
\hline \multirow{2}{*}{ P4 } & MCPA (250 mg/L) & 250 & 217 & $340^{*}$ & $176^{*}$ & 200 & 247 & 238 & 57 & 24 \\
& MCPP (150 mg/L) & 150 & 123 & $269^{*}$ & 120 & 137 & 138 & 156 & 56 & 36 \\
\hline
\end{tabular}

Valeurs cibles entre parenthèses. * : écart supérieur à $20 \%$.

Tableau IV : Résultats (mg/L) par laboratoire (LI à L6) et moyenne, écart-type et coefficient de variation (m, s, CV\%) des cas réels (P5 à P8).

\begin{tabular}{|c|c|c|c|c|c|c|c|c|c|c|}
\hline & & Li & $\mathrm{L} 2$ & $\mathbf{L 3}$ & L4 & L5 & L6 & $\mathbf{m}$ & s & $\mathrm{CV} \%$ \\
\hline P5 & Triclopyr (250 mg/L) & 260 & 231 & 255 & $160^{*}$ & 228 & 216 & 225 & 36 & 16 \\
\hline$P 6^{\circ}$ & $\begin{array}{l}\text { 2,4-D } \\
\text { MCPP }\end{array}$ & $\begin{array}{l}202 \\
760\end{array}$ & $\begin{array}{l}177 \\
670\end{array}$ & $\begin{array}{l}133^{*} \\
553\end{array}$ & $\begin{array}{l}196 \\
680\end{array}$ & $\begin{array}{l}141 \\
611\end{array}$ & $\begin{array}{l}202 \\
695\end{array}$ & $\begin{array}{l}175 \\
662\end{array}$ & $\begin{array}{l}31 \\
72\end{array}$ & $\begin{array}{l}18 \\
11\end{array}$ \\
\hline P7 & $\begin{array}{l}2,4-\mathrm{D}(155 \mathrm{mg} / \mathrm{L}) \\
2,4,5-\mathrm{T}(92 \mathrm{mg} / \mathrm{L})\end{array}$ & $\begin{array}{c}170 \\
95\end{array}$ & $\begin{array}{l}160 \\
104\end{array}$ & $\begin{array}{l}101 * \\
61^{*}\end{array}$ & $\begin{array}{l}173 \\
96\end{array}$ & $\begin{array}{l}135 \\
82\end{array}$ & $\begin{array}{c}168 \\
97\end{array}$ & $\begin{array}{c}151 \\
89\end{array}$ & $\begin{array}{l}28 \\
16\end{array}$ & $\begin{array}{l}19 \\
17\end{array}$ \\
\hline P8 & $\begin{array}{l}2,4-D(370 \mathrm{mg} / \mathrm{L}) \\
\operatorname{MCPA}(340 \mathrm{mg} / \mathrm{L})\end{array}$ & $\begin{array}{l}375 \\
400\end{array}$ & $\begin{array}{l}330 \\
321\end{array}$ & $\begin{array}{l}461 * \\
442 *\end{array}$ & $\begin{array}{l}328 \\
306\end{array}$ & $\begin{array}{c}276^{*} \\
300\end{array}$ & $\begin{array}{l}359 \\
349\end{array}$ & $\begin{array}{l}355 \\
353\end{array}$ & $\begin{array}{l}62 \\
57\end{array}$ & $\begin{array}{l}17 \\
16\end{array}$ \\
\hline
\end{tabular}

Valeurs initiales entre parenthèses. ${ }^{\circ}$ pool de 3 plasmas. * : écart supérieur à $20 \%$. 
vées : une estérification de ces produits sur la colonne lors de l'étape de lavage au méthanol est-elle possible ? Le pourcentage d'acide formique (2\%) dans le méthanol est-il suffisant pour éluer de la même façon tous les produits ? Cependant ce laboratoire ayant utilisé le MCPB comme étalon interne et par ailleurs les gammes d'étalonnage ayant été faites dans les mêmes conditions que les plasmas tests, ces différences restent difficiles à expliquer. Un nouvel essai a été effectué par ce même laboratoire, 5 mois plus tard, sur les mêmes échantillons, avec une colonne chromatographique neuve et des gammes d'étalonnage obtenues à partir de nouvelles poudres standards. Les résultats sont plus cohérents avec les valeurs attendues mais 3 concentrations ( 1 surcharge et 2 cas réels) sont encore en dehors de $\pm 20 \%$. La question reste donc pour le moment en suspens.

Deux laboratoires ont mentionné la présence dans les cas réels de 2 autres substances : le fluroxypyr ( 8 et 14 $\mathrm{mg} / \mathrm{L}$ ) dans l'échantillon $\mathrm{P} 5$ et le clopyralid (63 mg/L) dans l'échantillon P8. Le fluroxypyr est une molécule dérivée, comme le triclopyr, de l'acide pyridoxyacétique, le clopyralid étant dérivé de l'acide pyridoxy-2carboxylique. Le cas $n^{\circ} 5(H, 56$ ans) correspondait effectivement à une intoxication modérée par 5 à 6 verres de GARLON(B), mélange de triclopyr et fluroxypyr à 60 et $20 \mathrm{~g} / \mathrm{L}$ respectivement. Le prélèvement a été effectué environ à la $7^{\circ}$ heure après l'ingestion. Une intoxication modérée par un mélange 2,4-D et MCPP (cas n ${ }^{\circ} 6, \mathrm{H}, 42$ ans) a cependant montré des concentrations initiales (T2h) assez importantes et respectivement égales à 240 et $870 \mathrm{mg} / \mathrm{L}$. Mais l'évolution a été favorable sans doute en raison de la prise en charge précoce de ce patient en milieu hospitalier spécialisé. Le cas $n^{\circ} 7(\mathrm{H}, 36$ ans) est une intoxication au 2,4-D et 2,4,5- $\mathrm{T}$ avec une symptomatologie mineure et une prise en charge à la $3^{\circ}$ heure. Le cas $n^{\circ} 8(\mathrm{H}, 88$ ans) est une intoxication mortelle par $500 \mathrm{ml}$ de LONPARß, mélange de 2,4-D, MCPA et clopyralid respectivement à 150,175 et $35 \mathrm{~g} / \mathrm{L}(7)$. Les concentrations plasmatiques, égales à $700 \mathrm{mg} / \mathrm{L}$ pour le $2,4-\mathrm{D}$ et $750 \mathrm{mg} / \mathrm{L}$ pour le MCPA au moment de l'admission (T4h), étaient respectivement de 370 et $340 \mathrm{mg} / \mathrm{L}$ au moment du décès à la $26^{\circ}$ heure.

Enfin le laboratoire utilisant la spectrométrie de masse en tandem a signalé la présence de certains de ces phénoxyacides à l'état de «traces » $(<2 \mathrm{mg} / \mathrm{L})$ tant dans les plasmas surchargés, correspondant à des impuretés des produits purs, que dans les cas réels pouvant peut-être s'expliquer soit par des impuretés des produits techniques ingérés par les patients ou par une exposition de ceux-ci à ces produits en dehors d'un contexte aigu.

\section{Conclusion}

Dans cette étude orientée sur le diagnostic analytique des intoxications aiguës par herbicides phénoxyacides, plusieurs méthodologies ont été évaluées. La précipita- tion des protéines plasmatiques suivie d'une dilution au $1 / 2$ dans l'eau et d'une chromatographie avec détection UV et identification spectrale est une approche simple, rapide, nécessitant peu d'échantillon plasmatique et largement suffisante pour l'objectif défini. Dans les cas d'intoxication légère, le recours à une approche par extraction liquide/liquide, ou peut-être liquide/solide, d'un volume plus important de plasma devrait permettre d'abaisser la limite de détection. Cette approche devra être envisagée en cas d'exposition professionnelle et la spectrométrie de masse en tandem sera le meilleur mode de détection pour mesurer des concentrations faibles dans l'urine (de quelques $\mu \mathrm{g} / \mathrm{L}$ à 0,5 $\mathrm{mg} / \mathrm{L}$ ) de personnes exposées à ces substances lors d'applications agricoles (8-11).

\section{Références}

1. Fournier J. Identité chimique et activité biologique d'un produit. In : Fournier J., Chimie des pesticides. Lavoisier, Paris, 1988 : 95-109.

2. Poisindex, Micromedex Healthcare series, Chicago, 2006, Vol 127.

3. Flanagan RJ., Ruprah M. HPLC measurement of chlorophenoxy herbicides, bromoxynil, and ioxynil, in biological specimens to aid diagnosis of acute poisoning. Clin. Chem. $1989 ; 35: 1342-7$.

4. Van Damme JC., Galoux M. Méthode d'analyse par chromatographie liquide haute performance des formulations à base de mélange d'acides phénoxycarboxyliques, de dicamba, d'ioxynil et de bromoxynil. J. Chromatogr. $1980 ; 190: 401-10$.

5. Prescott LF., Park J., Darrien I. Treatment of severe 2,4-D and mecoprop intoxication with alkaline diuresis. Br. J. Clin. Pharmacol. $1979 ; 7: 111-6$.

6. Turcant A., Premel-Cabic A., Cailleux A., Allain P. Toxicological screening of drugs by microbore high-performance liquid chromatography with photodiode-array detection and UV spectral library searches. Clin. Chem. $1991 ; 37: 1210-5$.

7. Blanchet J.P., Turcant A., Harry P., Durand R., Allain P. Intoxication mortelle par le LONPAR. Ann. Tox. Anal. $2000 ; 12(2) 169$.

8. Kolmodin-Hedman B., Höglund S., Åkerblom M. Studies on phenoxy acid herbicides. I. Field study. Occupational exposure to phenoxy acid herbicides in agriculture. Arch. Toxicol. $1983 ; 54: 257-65$.

9. Kolmodin-Hedman B., Höglund S., Swensson Å., Åkerblom M. Studies on phenoxy acid herbicides. II. Oral and dermal uptake and elimination in urine of MCPA in humans. Arcl. Toxicol. 1983 ; 54 : 267-73.

10. Shealy D., Bonin M., Wooten J., Ashley D., Needham L. Application of an improved method for the analysis of pesticides and their metabolites in the urine of farmer applicators and their families. Environnement International $1996 ; 22: 661-75$.

11. Maroni M., Colosio C., Ferioli A., Fait A. Biological monitoring of pesticide exposure : a review. Toxicology $2000 ; 143: 1-118$. 